\title{
Sexual risk among pregnant women at risk of HIV infection in Cape Town, South Africa: What does alcohol have to do with it?
}

Amanda P. Miller, Department of Epidemiology, Fielding School of Public Health, University of California, Los Angeles, CA, USA

Steven Shoptaw, Department of Family Medicine, David Geffen School of Medicine, University of California, Los Angeles, CA, USA; Department of Psychiatry, University of Cape Town, Cape Town, South Africa.

Rufaro Mvududu, Division of Epidemiology and Biostatistics, School of Public Health and Family Medicine, University of Cape Town, Observatory, South Africa.

Nyiko Mashele, Division of Epidemiology and Biostatistics, School of Public Health and Family Medicine, University of Cape Town, Observatory, South Africa

Thomas J. Coates, Division of Infectious Diseases, David Geffen School of Medicine, University of California Los Angeles, Los Angeles, CA, USA

Linda-Gail Bekker, The Desmond Tutu HIV Centre, Institute for Infectious Disease and Molecular Medicine, Faculty of Health Science, University of Cape Town, Cape Town, South Africa

Zaynab Essack, Centre for Community-Based Research, Human Sciences Research Council, South Africa

Candice Groenewald, Centre for Community-Based Research, Human Sciences Research Council, South Africa; Honorary Research Associate, Psychology Department, Rhodes University, South Africa

Zaino Peterson, Impact and Research Development, Human Sciences Research Council, South Africa $^{9}$

Pamina M. Gorbach, Department of Epidemiology, Fielding School of Public Health, University of California, Los Angeles, CA, USA

Landon Myer, Division of Epidemiology and Biostatistics, School of Public Health and Family Medicine, University of Cape Town, Observatory, South Africa

Dvora L. Joseph Davey Department of Epidemiology, Fielding School of Public Health, University of California, Los Angeles, CA, USA; Division of Epidemiology and Biostatistics, School of Public Health and Family Medicine, University of Cape Town, Observatory, South Africa; Division of Infectious Diseases, David Geffen School of Medicine, University of California Los Angeles, Los Angeles, CA, USA; The Desmond Tutu HIV Centre, Institute for Infectious Disease and Molecular Medicine, Faculty of Health Science, University of Cape Town, Cape Town, South Africa 


\begin{abstract}
This study examines associations between alcohol use and HIV sexual risk among a cohort of HIV-uninfected pregnant women $(\mathrm{n}=1201)$ residing in a high HIV burden community in South Africa. Alcohol use was measured using a modified version of the Alcohol Use Disorder Identification Test (AUDIT). HIV sexual risk was measured through a composite variable of four risk factors: diagnosis with a STI, self-report of $>1$ recent sex partners, partner HIV serostatus (unknown or HIV+) and condomless sex at last sex. Any past year alcohol use prior to pregnancy was reported by half of participants (50\%); $6.0 \%$ reported alcohol use during pregnancy. Alcohol use prior to pregnancy was associated with increased odds of being at high risk of HIV $(\mathrm{aOR}=1.33$ for 2 risks and $\mathrm{aOR}=1.47$ for 3 risks $)$. In addition to reducing alcohol use, several other strategies to address HIV sexual risk in this population were identified.
\end{abstract}

Key words: alcohol use, pregnancy, HIV, South Africa, sub-Saharan Africa 


\section{Introduction}

Heavy alcohol use and HIV are prevalent interrelated public health issues that are associated with a substantial burden of disease in South Africa (1). With a national HIV prevalence of 19\%, South Africa is home to the most persons living with HIV globally (7.8 million) (2). Although only $31 \%$ of South Africa's population reports alcohol use in the past 12 months, consumption levels are high among those who drink, with 59\% engaging in heavy episodic drinking (HED), which is defined as the consumption of 60 grams or more of pure alcohol (the equivalent of six standard drinks) in one sitting (3). Estimates for total per capita consumption among drinkers (29.9 liters of pure alcohol), prevalence of alcohol use disorders (AUD) (7\%) and prevalence of alcohol dependence (2\%) in South Africa exceed regional averages (3). There are gendered differences in patterns of use and quantity of alcohol consumption in South Africa, with men being both more likely to drink and to consume hazardous levels of alcohol (3). However, HED is prevalent among women who consume alcohol. A recent report by World Health Organization (WHO) found that roughly one-third (34\%) of women reported having engaged in this behavior in the month preceding the survey (3) while another study found that a similar proportion of women (32\%) reportedly engage in binge drinking on a weekly basis (4). Alcohol use is also common among pregnant women in Cape Town (5-8). Prior work among pregnant women in this setting found that $29 \%$ reported alcohol use during pregnancy (9), an estimate that is likely biased due to underreporting/social desirability bias (10). Biomarker measures of hazardous alcohol use (23\%) have also confirmed use among pregnant women who are living with HIV in South Africa (11). 
Both alcohol use and incident HIV infection during pregnancy are associated with adverse health outcomes for the mother and fetus. Fetal alcohol spectrum disorder (FASD) describes a continuum of cognitive and physical impairments a fetus may experience due to prenatal alcohol exposure (PAE), the most severe form being fetal alcohol syndrome (FAS) (12). As a result of heavy alcohol use in pregnancy, South Africa has the highest rate of FAS in the world (13). FAS is a lifelong diagnosis and its deleterious effects on the central nervous system can result in neurocognitive developmental delays and behavioral problems that can persist into adolescence and young adulthood $(14,15)$. There is also evidence of socioeconomic disparities in the "harm per liter" of alcohol use, with poor South Africans experiencing worse outcomes associated with their alcohol use. Sixty percent of deaths attributable to alcohol use occur among persons in the bottom third of the socioeconomic spectrum (16). These socioeconomic disparities also extend to alcohol attributable HIV mortality (17). In addition to directly threatening the health of both mother and baby, alcohol use is a widely recognized driver of the HIV epidemic.

Alcohol use can impact decision making around sexual practices including having multiple sex partners $(18,19)$ and condomless sex $(18,20)$, resulting in increased risk of HIV acquisition $(21$ 24). Alcohol use is also associated with increased risk of intimate partner violence (IPV) (25, 26), which itself is a risk factor for HIV, through forced sex and reduced ability to safely negotiate condom use (27). While the evidence base supporting alcohol use as a risk factor for engaging in sexual risk behavior is well established (21), data examining this relationship among pregnant women in South Africa is more limited. Prior work with pregnant women found that alcohol use prior to (Cape Town (28)) or during (Mpumalanga (29)) pregnancy was associated with having multiple sexual partners but not condomless sex (29). In another Cape Town study 
one in four pregnant women $(27 \%)$ indicated that alcohol and drug use leads to greater sexual risk taking (30). Alcohol use is also associated with barriers to optimum health outcomes in the pre-exposure prophylaxis (PrEP) care continuum, around initiation and adherence (31), reducing the utility of the most effective biomedical intervention available to reduce HIV acquisition in individuals at high risk of infection. A robust body of literature suggests that alcohol use is also a barrier to ART uptake and adherence among persons living with HIV (32), including pregnant women (33). The effects of alcohol use on HIV risk, including non-adherence to PrEP, are especially problematic in pregnant women, due to elevated risk for HIV acquisition during pregnancy (34) as well as increased risk of vertical transmission due to high maternal viral load during the acute infection phase $(35,36)$. These intertwined public health issues of alcohol use, risky sexual practices, IPV and HIV can result in excess disease burden and exacerbate health disparities.

Addressing alcohol use among pregnant women is critical to HIV prevention efforts and public health efforts to promote healthy pregnancies and births. Given that alcohol use is a modifiable behavior, the importance of characterizing alcohol use, and identifying associations between alcohol and other HIV risk factors among pregnant women who experience a high burden of HIV incidence cannot be overlooked. This knowledge can inform the development of contextually sensitive interventions to interrupt and potentially prevent alcohol use during pregnancy. Given that withdrawal can be medically dangerous among persons experiencing alcohol dependence (for both mother and fetus) it is also important to identify women experiencing alcohol dependence in pregnancy to refer them to treatment centers that can facilitate supervised detoxification (37). The present analysis fills an important gap in the 
existing literature regarding alcohol use as a driver of sexual risk behavior among pregnant women by exploring associations between alcohol use and HIV sexual risk, among a large cohort of pregnant women residing in an underserved community experiencing a heavy burden of HIV in Cape Town, South Africa. We measure HIV sexual risk using a composite proxy risk measure to identify modifiable risk factors and hypothesize that alcohol use will be associated with greater odds of being in the high-risk category. We also examine sociodemographic and behavioral factors associated with alcohol use in this population.

\section{Methods and Materials}

\section{Participants and Procedures}

Pre-exposure prophylaxis in Pregnancy and Post-partum (PrEP-PP) is an ongoing observational prospective closed-cohort study of 1201 pregnant and postpartum women attending antenatal care (ANC) in Gugulethu, Cape Town, South Africa (clinical trial no. NCT03902418). This community was selected because of the high incidence and prevalence of HIV among pregnant and breastfeeding women (38). Consecutive eligible, consenting study participants were enrolled at the study site until the target sample size of 1201 pregnant women was met. Women 16 years or older attending their first ANC visit and pregnant at time of enrollment were eligible for study participation. Additional inclusion criteria included confirmed HIV negative serostatus (confirmed with $4^{\text {th }}$ generation ab/ag Abbott rapid test), intention to give birth in Cape Town, no medical or psychiatric conditions contraindicated for PrEP and consent to participate in the study. Participants are followed until 12 months' post-partum or until censorship (pregnancy loss, infant death, seroconversion, moving away, transfer out of care, loss to follow-up). Consented, enrolled participants are invited to return every 3 months for study visits that 
correspond with their ANC visits. All study staff are trained, salaried staff working for University of Cape Town.

The present analysis utilizes baseline data $(n=1,201)$ from study enrollment visits, collected between August 2019 and September 2021. Trained research staff conducted eligibility screening, HIV counseling and testing, counseling around HIV prevention and PrEP use, offer to initiate PrEP, diagnostic testing for other STIs and a counselor administered survey which takes approximately 30-45 minutes to complete. For STI testing and management, participants were instructed on how to do a self-collected vaginal swab which was tested for Chlamydia trachomatis (CT), Neisseria gonorrhoeae (NG), and Trichomonas vaginalis (TV) using point of care testing (Cephid, Inc., Sunnyvale, CA, US). Treatment is provided as needed during the same visit following South Africa National STI Guidelines (39). The study interviewers collect data on participant sociodemographics, sexual practices, depression, alcohol use, and attitudes and knowledge towards PrEP.

Written informed consent was obtained from all study participants; unassisted self-consent was obtained from adolescents (participants 16 and 17 years of age). The study received approval from UCT Human Research Ethics Committee (HREC) to waive parental consent. Participants were given the informed consent form to take home if they wanted to discuss with partners or parents and could return within 24 hours if they wanted to participate in the study. Participants viewed a video describing the study and the consent process in isiXhosa, the primary language spoken in this setting, to ensure that they understood the study design and consent form fully before signing. Light refreshments, compensation for their time (R100, USD\$7) as well as 
reimbursement for transportation are provided at each study visit (40). This study was approved by University of Cape Town Faculty of Health Sciences Human Research Ethics Committee (UCT-HREC) and University of California Los Angeles (UCLA).

\section{Measures}

Alcohol use, our exposure of interest, was measured using a modified versions of the 10 item Alcohol Use Disorders Identification Test (AUDIT) (41, 42), a validated measure that has been used globally, including among pregnant women in Cape Town $(43,44)$. The AUDIT typically asks questions for the reference period of "the past year". For the present study, questions were modified to the reference period "since finding out you were pregnant". The AUDIT is scored by summing responses to the 10 items (score range of 0-40 with high scores indicative of greater alcohol use) and a cutoff is used to differentiate between lower risk use, hazardous or harmful use and persons with probable alcohol dependence. A subset of the AUDIT, the first three AUDIT questions which are used to measure alcohol consumption levels (AUDIT-C (45)), were used to identify hazardous alcohol use in the year prior to pregnancy (range 0-12). AUDIT-C questions were also asked for the reference period, "in the past year prior to finding out you were pregnant". Given the adverse consequences of any amount of alcohol use while pregnant on neonatal outcomes, the potential for underreporting of alcohol use while pregnant, and prior work which suggests that alcohol use prior to pregnancy is a good predictor of alcohol use during pregnancy (46), our primary alcohol exposure of interest was any alcohol use prior to pregnancy (an AUDIT-C score of 0). Limited data on patterns of alcohol use in South Africa immediately prior to and during pregnancy exist; to address this gap, we also explored patterns of risky alcohol use prior to and during pregnancy as secondary exposures of interest. We consider all 
alcohol use during pregnancy to be "risky"; therefore, we adopted a conservative AUDIT cutoff of $\geq 5$ to capture even light to moderate patterns alcohol use which we would consider "higher risk" during pregnancy. This "higher risk" cutoff captures the categories of risky, harmful, and severe alcohol use in women and has previously been used among pregnant women in South Africa (43). To identify women with probable alcohol dependence, we used a cut-off of $\geq 20$. For the AUDIT-C we used a cut-off of $\geq 3$ for hazardous drinking, a cut-off previously used with pregnant women in South Africa (11).

HIV risk, our primary dependent variables of interest, was measured through a proxy composite variable of four risk factors: (1) diagnosis with a STI infection (measured at baseline), (2) selfreport of multiple sexual partners (reporting more than one partner in the past 3 months), (3) selfreport of partner HIV serostatus (dichotomized into two categories: HIV-negative/no partner and HIV-positive/unknown serostatus), and (4) self-report of condomless sex at last sex. An ordinal count variable was created for individuals experiencing these risk factors. A sensitivity analysis was performed to identity an optimum cutoff and a binary composite HIV sexual risk variable was created for those at high-risk vs not high-risk. Results are presented for two cut offs: we explore classifying women who experience two or more HIV risk factors as high risk as well as classifying women who experience three or more HIV risk factors as high risk.

Other measures analyzed in descriptive and multivariable analysis included sociodemographic variables such as age, educational attainment (did not complete secondary school/completed secondary school), employment status (a six category variable collapsed into a three category variable: any form of employment/unemployed/student), residence type (a five category variable 
collapsed into a binary variable: formal/informal housing) and beliefs that may impact alcohol use during pregnancy such as participants' pregnancy intentions, which were measured using items adapted from prior studies of fertility intentions in this setting $(47,48)$, including feelings about the timing of the pregnancy (did this pregnancy happen at the right/wrong time?) and having a baby (I wanted to have a baby/I have mixed feelings about having a baby/I did not want to have a baby). HIV risk factors were also considered, including current sexual partner (no/yes, the father of my unborn baby/yes someone else), relationship status (cohabitating/not cohabitating), likelihood their partner has other sexual partners (not likely at all/ somewhat likely/very likely/I don't know) and frequency of condom use in past 3 months (never/rarely/sometimes/almost always/always). Other risk factors included depression, using the Edenborough Post-natal Depression Scale (EPDS) (49) (cutoff of $\geq 11$ for depression (50)) and experiences of past year verbal, physical and sexual IPV using 13 items adapted from the WHO IPV Scale (collapsed into a binary variable: any/no recent experiences of IPV) $(51,52)$.

\section{Data Analysis}

Analyses were conducted using SAS studio (53). The data were first inspected for errors, omissions, and values lying outside of the limit ranges. In order to identify the most appropriate alcohol use measure to use in the present analysis, a sensitivity analysis was performed. The analytic sample was restricted to participants who provided responses to our primary exposure of interest, alcohol use, resulting in an analytic sample of 1201. The distribution, mean and median, for each of our alcohol measures were explored as well as the internal reliability (Cronbach's alpha coefficient) and cut-off points were informed by the existing literature. Prior research has found that alcohol use prior to pregnancy is a good predictor of alcohol use in pregnancy (46). It 
is also worth noting that prior work in this study sample found a median gestational age at baseline (first ANC visit) of 21 weeks (the fifth month of pregnancy) (54), so "prior to pregnancy" may encompass the entire first trimester, a critical time in fetal development. Based on this sensitivity analysis and our prior work it was determined that past year alcohol use prior to pregnancy would be the alcohol measure used in bivariate and multivariable analyses. Next, sociodemographic and HIV risk related behavioral variables of interest were analyzed, using descriptive statistics, to characterize the analytic sample, overall, and by alcohol use. Stratified bivariate analysis of covariates by any past year alcohol use prior to pregnancy was performed using $\chi 2$ analysis, fisher's exact test and two sample t-test. Statistical significance was determined using an alpha of 0.05 .

To test our two hypotheses, multivariable logistic regression models were built using the proc logistic function and the logit link to explore associations between past year alcohol use prior to pregnancy and our HIV risk outcome. Directed acyclic graphs were used a priori to determine the minimally sufficient set of covariates to adjust for to reduce bias from measured confounders (see supplemental file). A variable was considered a confounder if it was predictive of both the exposure and outcome and was not on the causal pathway between exposure and outcome. Multicollinearity was assessed by examining the intercorrelations between the exposure variables in the model as well as the tolerance and variance inflation factor (VIF). All VIF approximated 1, no tolerance values were $<0.1$ and no correlations exceeded 0.7 .

\section{$\underline{\text { Results }}$}




\section{Description of the study sample}

Table 1 provides relevant sociodemographic and behavioral characteristics from baseline visits (i.e., first ANC appointment) for the 1201 study participants. Characteristics are provided for the overall sample and stratified by past year alcohol use prior to pregnancy. Mean age of participants was 26.6 years (SD 5.9); roughly half $(51 \%, \mathrm{n}=617)$ had completed secondary schooling and resided in an informal dwelling (54\%, $\mathrm{n}=644)$. The median gestational age was 21 weeks (IQR 15-31). Half $(50 \%, n=598)$ felt the timing of the pregnancy was wrong and nearly half $(49 \%, n=585)$ reported they did not want to have the baby. Past year IPV was reported by $12 \%(n=147)$ of participants and 7\% $(n=89)$ of participants had EPDS scores indicative of current depression. Most participants currently had a sexual partner $(92 \%, \mathrm{n}=1,105)$ and this partner was almost always the baby's father. Nearly one third of participants $(29 \%, \mathrm{n}=345)$ were diagnosed with a STI infection; more than one in five participants $(22 \%, \mathrm{n}=268)$ were unaware of their partners HIV serostatus while 2\% $(n=18)$ reporting having a partner living with HIV. Most participants $(69 \%, \mathrm{n}=804)$ reported never using a condom in the prior three months. Mean age, residence type, past year IPV, having multiple sexual partners, pregnancy timing, feelings about the pregnancy, cohabitation and primary partner's HIV status significantly differed by alcohol use status. Persons reporting alcohol use (versus no alcohol use) were more likely to be younger (mean age: 25.9 years vs 27.3 years), less likely to feel the pregnancy timing was good ( $45 \%$ vs $55 \%$ ), less likely to report wanting to have the baby (30\% vs $42 \%$ ), more likely to have a partner of unknown HIV serostatus (25\% vs $19 \%)$, more likely to report IPV (17\% vs $8 \%$ ) and more likely to have multiple partners ( $5 \%$ vs $1 \%)$. 
Results from the sensitivity analysis of our alcohol use measures before and during pregnancy can be found in Table 2. Any alcohol use in the past year prior to pregnancy was reported by half of participants $(50 \%, \mathrm{n}=598)$. Prior to pregnancy, one-third $(33 \%, \mathrm{n}=396)$ of the sample engaged in hazardous drinking; when looking exclusively among those reporting any alcohol use during this time frame, the majority $(66 \%, n=396)$ engaged in hazardous drinking. Six percent of participants reported alcohol use during pregnancy. Of those reporting any alcohol use during this time frame, using a conservative AUDIT cutoff to capture the categories of risky, harmful and severe alcohol use, $46 \%(\mathrm{n}=33)$ of pregnant women reporting any alcohol use during pregnancy had AUDIT scores indicative of higher risk alcohol use. One third $(32 \%, \mathrm{n}=23)$ of pregnant women reporting any alcohol use during pregnancy had AUDIT-C scores indicative of heavy alcohol use. Three percent of women reporting any alcohol use during pregnancy had an AUDIT score indictive of probable alcohol dependence. The AUDIT and AUDIT-C (for both reference periods: during and before pregnancy) had Cronbach's alpha coefficients suggestive of good internal consistency $(>0.8)$ in our study sample.

\section{Descriptive characteristics of women at highest risk of HIV}

Table 3 presents sociodemographic characteristics of women in the highest risk factor (3+ risks) group (n=94). Among women with three of more HIV risks factors, mean age of participants was 25.8 years $(\mathrm{SD} 6.3) ; 38 \%(\mathrm{n}=36)$ had completed secondary schooling and $62 \%(\mathrm{n}=58)$ resided in an informal dwelling. Fifty nine percent $(n=55)$ reported alcohol use prior to pregnancy while $9 \%(\mathrm{n}=8)$ reported alcohol use during pregnancy. The majority $(58 \%, \mathrm{n}=54)$ felt the timing of the pregnancy was wrong and more than half $(56 \%, \mathrm{n}=53)$ reported they did not want to have the baby. Past year IPV was reported by $14 \%(n=13)$ of participants and $5 \%(n=5)$ of participants 
had EPDS scores indicative of depression. Nearly all participants currently had a sexual partner (99\%, $\mathrm{n}=93)$ and this partner was almost always the baby's father. The most common risk factor was condomless sex at last sex, reported by $99 \%(n=92)$ of participants, followed by a partner of positive or unknown HIV serostatus $(97 \%, \mathrm{n}=91)$, current STI infection $(93 \%, \mathrm{n}=87)$ and $15 \%$ $(n=14)$ had multiple sexual partners in the past 3 months. Three percent of women $(n=3)$ in this high-risk category had all four HIV risk factors.

\section{Bivariate and multivariable regression analysis of alcohol use on HIV risk}

Figure 1 presents the results from bivariate and multivariable analysis of the association between past year alcohol use prior to pregnancy and HIV risk. In multivariable analysis, the model was adjusted for the following potential confounders: age, education level, employment status, residence type and current partner. Using the cutoff of two or more risk factors to denote "high risk" for HIV acquisition, after adjusting for covariates, persons reporting past year alcohol use prior to pregnancy had 1.33 times greater odds of being in the high HIV risk category than persons reporting no alcohol use during that same time frame (adjusted OR 1.33, 95\% CI 1.051.68). Using the cutoff of three or more risk factors to denote "high risk" after adjusting for these covariates, persons reporting past year alcohol use prior to pregnancy had 1.47 times greater odds of being in the high HIV risk category than persons reporting no alcohol use during that same time frame (adjusted OR 1.47, 95\% CI 0.95-2.27).

\section{$\underline{\text { Discussion }}$}

Using multiple validated measures of alcohol use and cutoffs consistent with the existing literature, the present study fills important gaps in the literature regarding the potential role 
alcohol may play as a risk factor for engaging in sexual risk behaviors in pregnancy by exploring associations between alcohol use and HIV sexual risk among HIV-uninfected pregnant women in South Africa. Obtaining accurate estimates of alcohol use during pregnancy presents challenges. Alcohol use during pregnancy is stigmatized and subject to underreporting due to social desirability bias (55). In our study sample, as expected the prevalence of alcohol use was much lower during pregnancy relative to prior to pregnancy (6\% vs 50\%). Nevertheless, the prevalence of alcohol use prior to pregnancy in our sample was higher than that observed in another recent study of HIV-uninfected women in Cape Town (50\% vs 40\%), but the prevalence of heavy alcohol use (defined as an AUDIT-C score $\geq 3$ ) among those who reported any alcohol use, was similar in both samples at (66\% vs 65\%) (5). Observing a reduction of alcohol use and increased abstinence among pregnant women was expected; a large global body of evidence suggests that many women reduce their alcohol use or stop drinking entirely once their pregnancy is recognized (56-59). However, our estimate for alcohol use during pregnancy is lower than expected given estimates from prior studies. A recent systematic review and meta-analysis of alcohol use during pregnancy in sub-Saharan Africa found a pooled alcohol use during pregnancy prevalence of $24 \%$ (95\% CI 22.48, 24.60) for the nine studies from South Africa (60). Underreporting of alcohol use during pregnancy in our sample may partially account for the low prevalence observed; timing of recognition of pregnancy may have also influenced this measure.

Women in our study who reported alcohol use prior to pregnancy differed in several ways from women who did not report this behavior. They had increased odds of reporting recent IPV, consistent with a prior study among women and men attending drinking establishments in Cape Town that found associations between IPV and both alcohol consumption and binge drinking 
among pregnant women (61). Pregnant women who drank alcohol also had increased odds of being depressed and reporting an unplanned pregnancy, consistent with findings from a metaanalysis where depression and unplanned pregnancy were both predictors of alcohol use during pregnancy (60). While it is known that pregnant women, including women in South Africa, experience stigma around alcohol use during pregnancy from healthcare providers (62-65), there is a dearth of literature exploring community norms and attitudes towards maternal drinking and how these may facilitate alcohol use in this population (63). Some qualitative work has been undertaken to explore pregnant women's perceptions of these norms and attitudes (66), but there is a need for qualitative work with drinking partners, peers and family members to understand interpersonal and familial drivers of alcohol use during pregnancy and post-partum period.

Two recent systematic reviews of psychosocial interventions to address alcohol use in subSaharan Africa highlight the limited number of evidence-based interventions to address alcohol use in pregnancy in this setting $(67,68)$. The reviews found a total of two interventions $(69-71)$ aimed at addressing alcohol use during pregnancy, both in South Africa. A brief intervention (BI) produced a significant reduction in mean AUDIT score immediately after intervention delivery but did not report outcomes in the post-partum period (71). A peer-led mentor mother intervention providing a maternal care package (which included HIV prevention content and a single session on alcohol use) found increased abstinence 5 years postpartum but did not improve abstinence relative to the control group during the critical pregnancy and breastfeeding periods (70). Neither intervention was designed to address alcohol use among persons experiencing alcohol dependence. Resources to address alcohol and other substance dependence in South Africa are limited and socioeconomic inequities in access to such services exist (72). The disease 
burden associated with alcohol use and HIV during pregnancy and the dearth of available interventions to address these issues underscore the urgent need for culturally acceptable evidence-based interventions to address alcohol use (including alcohol dependence) and HIV prevention among pregnant women in South Africa.

The women in our study are at high risk of HIV infection generally, due to the high prevalence of HIV in their community and increased risk of HIV acquisition during pregnancy (34). Using both cut-offs $(2+$ risks and $3+$ risks $)$ we observed an effect in the expected direction with women reporting alcohol use having greater odds of being in the high HIV sexual risk category. The four risk factors including, partner HIV serostatus, having $>1$ recent sex partners, condomless sex at last sex and the presence of an STI, present opportunities for intervention. While the prevalence of having an HIV positive partner was low (2\%) more than one-fifth of the women in our study (22\%) were not aware of their partner's HIV serostatus. When looking at women in our high-risk group, 92\% were not aware of their partner's status. In the last decade, HIV self-testing (HIVST) (73) and couples HIV testing have emerged as promising interventions for reaching people that would otherwise be unlikely to access testing, such as male partners $(74,75)$.

The high prevalence of persons with partners of unknown HIV serostatus and high risk of HIV acquisition in pregnancy underscore the importance of integrating PrEP as a female controlled option for protection against HIV acquisition and onward transmission in this population. When taken correctly and consistently, daily oral PrEP (TDF/FTC) is a safe and highly effective method of HIV prevention (76-78). Further understanding of the relationship between alcohol 
use and PrEP initiation and adherence is needed to determine if alcohol is a barrier to optimal uptake of this HIV prevention measure in this population.

The prevalence of other STIs was also high in our study sample (29\%). While the prevalence of STIs was slightly higher among persons who use alcohol, this difference was not statistically significant. However, the high prevalence throughout the study sample makes addressing STIs in this population a public health priority. To avoid reinfection in these women, it is important that they disclose their status to their partner and encourage their partner to receive treatment. Qualitative work with HIV positive pregnant women in Pretoria highlights barriers and facilitators to partner disclosure of STI results that pregnant women experience; many participants indicated that fear of IPV was a barrier to disclosure (79). To avoid re-infection, interventions that support partner disclosure, expedited partner therapy and offer support services for women experiencing IPV are needed in addition to STI testing services (80).

Our study had several limitations. Data were cross-sectional, precluding our ability to infer directionality or causality and all our alcohol use and HIV sexual risk variables (except for STI testing) were self-reported and subject to underreporting due to social desirability bias. Our study also had several strengths; we used globally validated alcohol measures in a large sample of HIV uninfected pregnant women to provide timely estimates of the prevalence of alcohol use and other HIV sexual risk factors in a population at high risk of HIV infection.

\section{Conclusion}


We found a high prevalence of alcohol use prior to pregnancy in our study sample which may reflect high levels of alcohol use in early pregnancy. Furthermore, alcohol use was associated with being at high risk of HIV acquisition in HIV-uninfected pregnant women. Evidence-based interventions to address alcohol use and other HIV risk behaviors during pregnancy in South Africa are desperately needed. We identified several modifiable behaviors that could be targeted in future interventions including, improved measures to increase partner HIV testing and disclosure, and testing/counseling around STI results disclosure which could be integrated into ANC service delivery. Future studies should use objective measures of alcohol use as well as self-reported measures collected in real time to reduce bias from recall and social desirability. Finally, qualitative work is needed to understand individual and community level drivers of alcohol use among pregnant and breastfeeding women in this setting to develop a culturally tailored intervention to address these issues in this population. 
Table 1. Sociodemographic and behavioral associated with alcohol use in 12 months prior to pregnancy among pregnant women at risk of HIV in Cape Town, South Africa ( $n=1201$ women)

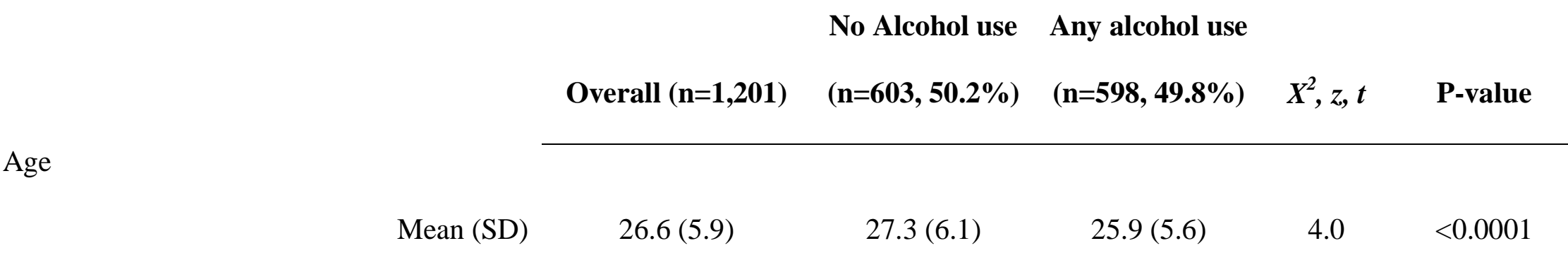

Gestational Age at

First ANC Visit

Median [IQR $\quad 21[15-31] \quad 22[15-32] \quad 21[14-30] \quad-0.6 \quad 0.5370$

Educational Attainment

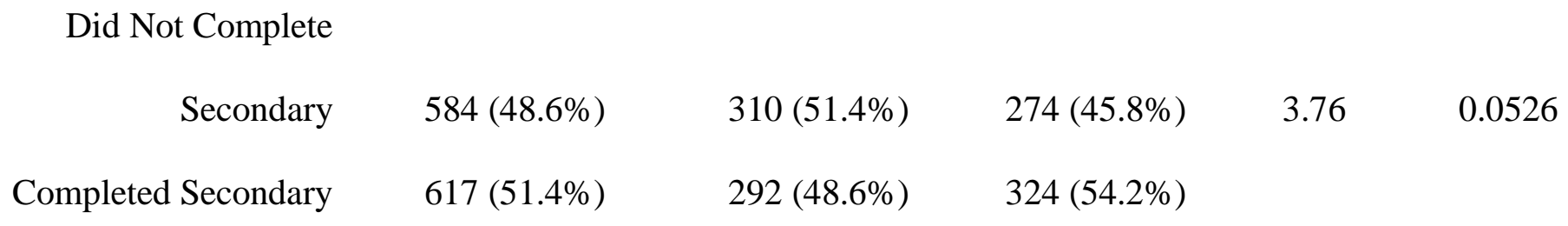


Employed (full/part-

$\begin{array}{rlll}\text { time, formal/informal) } & 431(35.9 \%) & 220(36.5 \%) & 211(35.3 \%) \\ \text { Student } & 131(10.9 \%) & 61(10.1 \%) & 70(11.7 \%) \\ \text { Unemployed } & 639(53.2 \%) & 322(53.4 \%) & 317(53.0 \%)\end{array}$

Residence Type

$16.1<0.0001$

$\begin{array}{cccc}\text { Informal Dwelling } & 644(53.6 \%) & 358(59.4 \%) & 250(47.8 \%) \\ \text { Formal Dwelling } & 557(46.4 \%) & 245(40.6 \%) & 312(52.2 \%)\end{array}$

Past year IPV

Depression

$\begin{array}{lrrr}\text { Yes } & 147(12.2 \%) & 45(7.5 \%) & 102(17.1 \%) \\ \text { No } & 1054(87.8 \%) & 558(92.5 \%) & 496(82.9 \%)\end{array}$

$$
557(46.4 \%)
$$

$245(40.6 \%)$

25.7

$<0.0001$

3.7 


$$
\text { No Depression } \quad 1112(92.6 \%) \quad 567(94.0 \%) \quad 545(91.4 \%)
$$

Pregnancy Timing

$12.2 \quad 0.0005$

Pregnancy happened

at the right time $\quad 603(50.2 \%) \quad 333(55.2 \%) \quad 270(45.2 \%)$

Pregnancy happened

at the wrong time

$598(49.8 \%)$

$270(44.8 \%)$

$328(54.9 \%)$

Feelings about pregnancy

$\begin{array}{ll}17.0 & 0.0002\end{array}$

I wanted to have a

$$
\text { baby } \quad 432(36.0 \%) \quad 251(41.6 \%) \quad 181(30.3 \%)
$$

I have mixed feelings

about having a baby

$194(15.3 \%)$

$87(14.4 \%)$

$97(16.2 \%)$

I did not want to have

$$
\text { a baby }
$$

$265(44.0 \%)$

$320(53.5 \%)$

Current Partner

$3.1 \quad 0.2136$

$\begin{array}{lll}\text { No partner } \quad 96(8.0 \%) & 54(9.0 \%) & 42(7.0 \%)\end{array}$




$$
\begin{aligned}
& \text { Yes, the partner is my } \\
& \text { unborn baby's father } \\
& 1095(91.2 \%) \\
& 546(90.1 \%) \\
& 549(91.8 \%) \\
& \text { father } \\
& 10(0.8 \%) \\
& 3(0.5 \%) \\
& 7(1.2 \%)
\end{aligned}
$$

Relationship Status

$(\mathrm{n}=1105)$

\author{
Cohabitating
}

Not Cohabitating

Primary Partner's HIV status

$\begin{array}{rccc}\text { Positive } & 20(1.7 \%) & 9(1.5 \%) & 11(1.8 \%) \\ \text { Negative } & 852(70.9 \%) & 450(74.6 \%) & 402(67.2 \%) \\ \text { Don't know } & 268(22.3 \%) & 116(19.2 \%) & 152(25.4 \%) \\ \text { No Recent Partner } & 61(5.1 \%) & 28(4.6 \%) & 33(5.5 \%)\end{array}$

Multiple partners in past 3 months
27.7

$<0.0001$

$8.1 \quad 0.0434$

$\begin{array}{lll}443(40.1 \%) & 263(47.9 \%) & 180(32.4 \%) \\ 662(59.9 \%) & 286(52.1 \%) & 376(67.6 \%)\end{array}$

$14.0 \quad 0.0002$ 


$\begin{array}{cccc}\text { Yes } & 36(3.0 \%) & 7(1.2 \%) & 29(4.9 \%) \\ \text { No } & 1071(96.8 \%) & 596(98.8 \%) & 569(95.2 \%)\end{array}$

Partner has other sexual partners $(n=1,095)$

$2.3 \quad 0.5052$

$\begin{array}{rlll}\text { Not likely } & 219(20.0 \%) & 110(20.2 \%) & 109(19.9 \%) \\ \text { Somewhat likely } & 423(38.6 \%) & 210(38.5 \%) & 213(38.8 \%) \\ \text { Very likely } & 166(15.2 \%) & 75(13.7 \%) & 91(16.6 \%) \\ \text { Don't know } & 287(26.2 \%) & 151(27.7 \%) & 136(24.8 \%)\end{array}$

Frequency of condom use in past 3 months $(n=1,167)$

$\begin{array}{rccc}\text { Never } & 804(68.9 \%) & 387(66.4 \%) & 417(71.4 \%) \\ \text { Rarely/Sometimes } & 317(27.2 \%) & 168(28.8 \%) & 149(25.5 \%) \\ \text { Almost } & & & \\ \text { always/Always } & 46(3.9 \%) & 28(4.8 \%) & 18(3.1 \%)\end{array}$

$4.4 \quad 0.10916$

Current STI diagnosis

$\begin{array}{llll}\text { Yes } & 345(28.7 \%) & 167(27.7 \%) & 178(29.8 \%) \\ \text { No } & 856(71.3 \%) & 399(71.1 \%) & 386(67.9 \%)\end{array}$


Composite HIV sexual risk measure

\begin{tabular}{rccc} 
0 risk factor & $74(6.2 \%)$ & $50(8.3 \%)$ & $24(4.0 \%)$ \\
1 risk factor & $625(52.0 \%)$ & $324(53.7 \%)$ & $301(50.3 \%)$ \\
2 risk factors & $408(334.0 \%)$ & $190(31.5 \%)$ & $218(36.5 \%)$ \\
3+ risk factors & $94(7.8 \%)$ & $39(6.5 \%)$ & $55(9.2 \%)$ \\
\hline
\end{tabular}

$14.6 \quad 0.0022$ 
Table 2. Performance of Alcohol Use Measures in the Study Sample Among Pregnant Women in Cape Town, South Africa $(\mathrm{n}=1,201)$

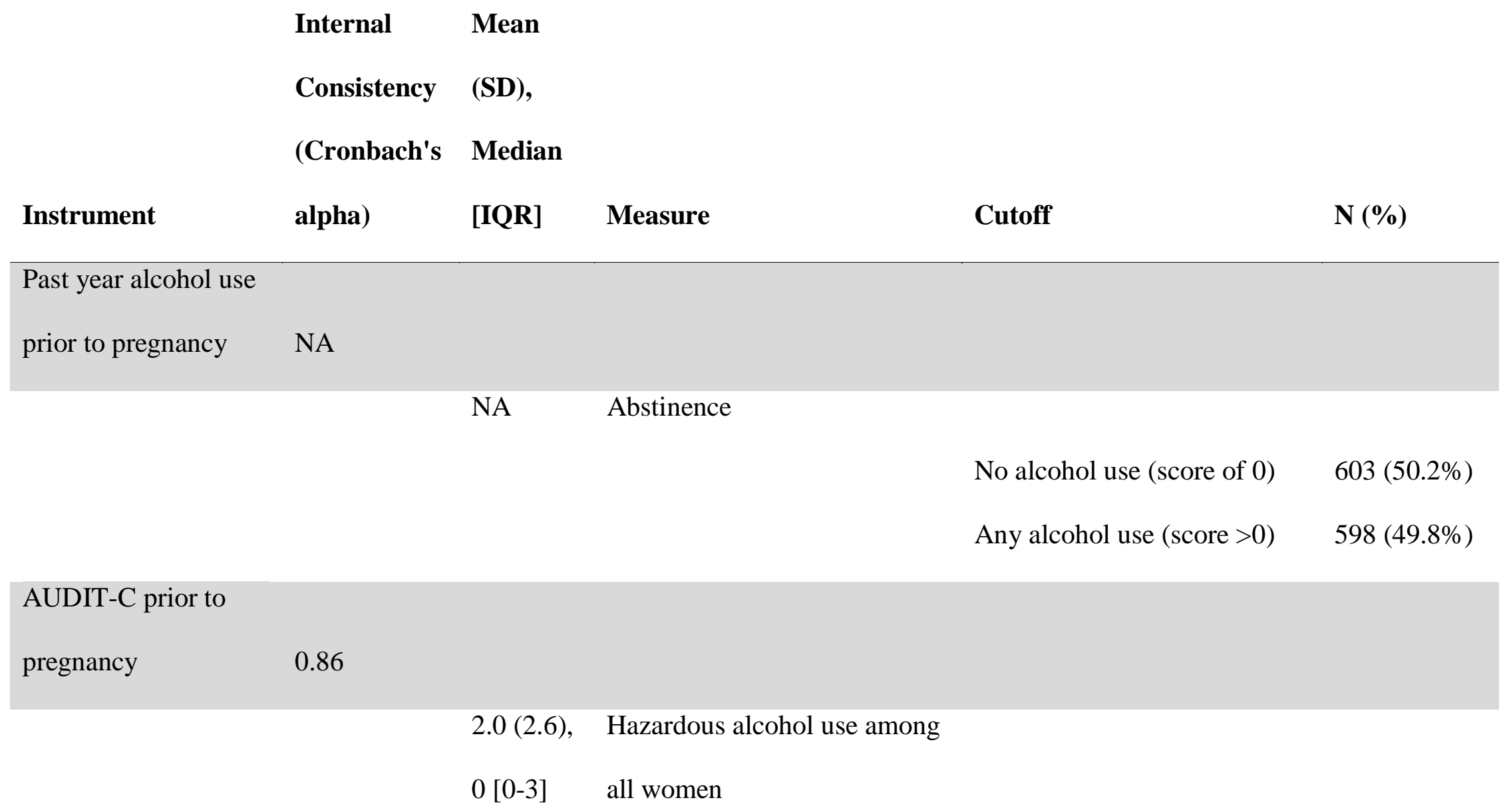


4.0 (2.4), Hazardous alcohol use among

3.0 [2-6] women reporting alcohol use

\section{Hazardous drinking $(\geq 3) \quad 396(66.2 \%)$}

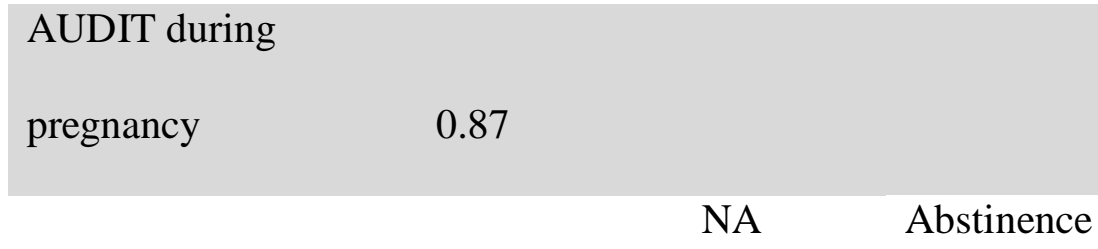

No alcohol use (score of 0 )

$(94.0 \%)$

Any alcohol use (score $>0$ )

$72(6.0 \%)$

0.3 (1.8), Higher risk alcohol use among

$0,[0,0]$ all women

High risk alcohol use (score

$<5)$

Higher risk alcohol use (score
1168

$(97.3 \%)$

$33(2.8 \%)$ 
5.6 (5.0), Higher risk alcohol use among

4 [2-8] women reporting alcohol use

High risk alcohol use (score

$39(54.2 \%)$

Higher risk alcohol use (score

$33(45.8 \%)$

5.6 (5.0), Alcohol dependence among

4 [2-8] women reporting alcohol use

Dependence not likely (score

$70(97.2 \%)$

Probable dependence (score

\section{AUDIT-C during}

pregnancy

0.83

0.2 (0.8), Heavy alcohol use among all 
0 [0-0] women

1178

Non-heavy alcohol use (<3) $\quad$ (98.1\%)

Heavy alcohol use $(\geq 3)$

$23(1.9 \%)$

2.5 (2.1), Heavy alcohol use among

2.0 [1-3] women reporting alcohol use 
Table 3. Sociodemographic and behavioral characteristics of women at high risk of HIV (using cutoff of $3+$ risk factors) $(n=94)$

$$
\mathbf{N}(\%)
$$

Age

$$
\text { Mean (SD) } \quad 25.8(6.3)
$$

Educational Attainment

$\begin{array}{rr}\text { Did not complete } & \\ \text { secondary } & 58(61.7 \%) \\ \text { Completed Secondary } & 36(38.3 \%)\end{array}$

Employment Status

Employed (full/part-

time, formal/informal)

$\begin{array}{rr}\text { Student } & 13(13.8 \%) \\ \text { Unemployed } & 53(56.4 \%)\end{array}$

Residence Type

$$
\begin{array}{cc}
\text { Informal Dwelling } & 58(61.7 \%) \\
\text { Formal Dwelling } & 36(38.3 \%)
\end{array}
$$

Past year IPV

$\begin{array}{ll}\text { Yes } & 13(13.8 \%) \\ \text { No } & 81(86.2 \%)\end{array}$


Any alcohol use before

pregnancy

Yes

No

Yes

No

Depression

Pregnancy Timing

Feelings about pregnancy
Depression (EPDS

$$
>=11)
$$

No Depression

Pregnancy happened at the right time

Pregnancy happened at $54(57.5 \%)$

the wrong time
$89(94.7 \%)$

$40(42.6 \%)$
$5(5.3 \%)$

I wanted to have a baby $30(31.9 \%)$

I have mixed feelings $\quad 11(11.7 \%)$ 


\section{Current Partner}

about having a baby

I did not want to have a

$53(56.4 \%)$

baby

No partner

Yes, the partner is my unborn baby's father

Yes, the partner is not my unborn baby's

father

Relationship Status (n=93)

Primary Partner's HIV status

Cohabitating

Not Cohabitating

$1(1.1 \%)$

$21(22.6 \%)$

$72(77.4 \%)$

$2(2.1 \%)$

$5(5.3 \%)$

Negative

$2(2.1 \%)$

Don't know

$86(91.5 \%)$

No Recent Partner

$1(1.1 \%)$

Multiple partners in past 3 months

$\begin{array}{ll}\text { Yes } & 14(14.9 \%) \\ \text { No } & 80(85.1 \%)\end{array}$


Partner has other sexual partners $(\mathrm{n}=91)$

$\begin{array}{rc}\text { Not likely } & 8(8.8 \%) \\ \text { Somewhat likely } & 33(36.3 \%) \\ \text { Very likely } & 23(25.3 \%) \\ \text { Don't know } & 27(29.7 \%)\end{array}$

Frequency of condom use in past 3 months

$\begin{array}{cc}\text { Never } & 73(77.7 \%) \\ \text { Rarely/Sometimes } & 21(22.3 \%) \\ \text { most always/Always } & 0(0.0 \%)\end{array}$

\section{Current STI infection}

Yes

$87(92.6 \%)$

No

$7(7.5 \%)$ 


\section{Figure 1. Unadjusted and adjusted ${ }^{1}$ analyses of associations between any alcohol use before pregnancy and HIV sexual risk $^{2}$}

HIV sexual risk level using $2+$ risks

(unadjusted)

HIV sexual risk level using 2+ risks (adjusted)

HIV sexual risk level using 3+ risks (unadjusted)

HIV sexual risk level using 3+ risks (adjusted)

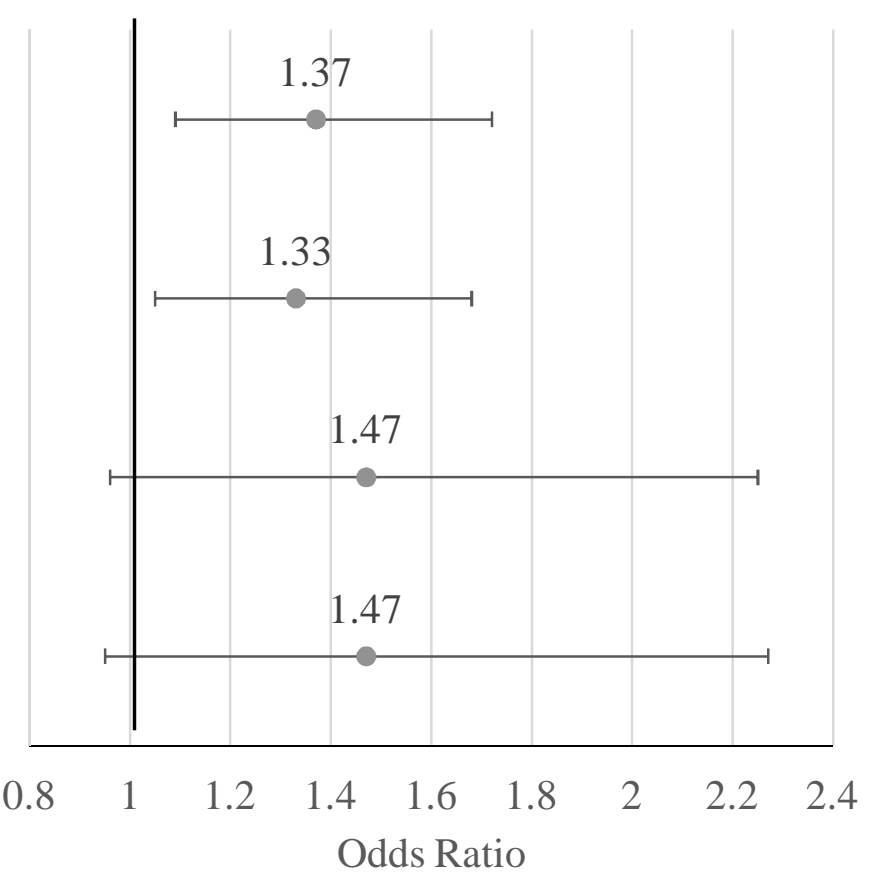

\footnotetext{
${ }^{1}$ Model adjusted for age, education level, employment status, residence type and current partner

${ }^{2}$ HIV sexual risk measured by presence of an STI, multiple partners, condom use at last sex and partner HIV status. Individuals with three or more of these risks were considered "high risk"
} 
Work Cited

1. IHME. South Africa Country Profile Seattle, WA.: Institute for Health Metrics and Evaluation; 2020 [Available from: http://www.healthdata.org/south-africa.

2. UNAIDS. South Africa Country Profile. 2020.

3. WHO. Global status report on alcohol and health 2018: Country Profiles for WHO Regions. Geneva, Switzerland: World Health Organization; 2018.

4. Bhana A, Groenewald C. Patterns of alcohol consumption in South Africa: Implications of harmful drinking for families. In: Mokomane Z, Roberts B, Struwig J, Gordon S, editors. South African Social Attitudes: Family Matters; family cohesion, values and wellbeing. South Africa: HSRC Press.; 2019.

5. Brittain K, Remien RH, Phillips T, Zerbe A, Abrams EJ, Myer L, et al. Factors associated with alcohol use prior to and during pregnancy among HIV-infected pregnant women in Cape Town, South Africa. Drug Alcohol Depend. 2017;173:69-77.

6. WHO. Global status report on alcohol and health 2018. Geneva, Switzerland.: World Health Organization, ; 2018.

7. Petersen Williams P, Jordaan E, Mathews C, Lombard C, Parry CD. Alcohol and Other Drug Use during Pregnancy among Women Attending Midwife Obstetric Units in the Cape Metropole, South Africa. Adv Prev Med. 2014;2014:871427.

8. Joseph Davey D, Farley E, Towriss C, Gomba Y, Bekker LG, Gorbach P, et al. Risk perception and sex behaviour in pregnancy and breastfeeding in high HIV prevalence settings: Programmatic implications for PrEP delivery. PloS one. 2018;13(5):e0197143. 
9. Joseph Davey D, Farley E, Gomba Y, Coates T, Myer L. Sexual risk during pregnancy and postpartum periods among HIV-infected and -uninfected South African women: Implications for primary and secondary HIV prevention interventions. PloS one. 2018;13(3):e0192982.

10. Del Boca FK, Darkes J. The validity of self-reports of alcohol consumption: state of the science and challenges for research. Addiction. 2003;98 Suppl 2:1-12.

11. Raggio GA, Psaros C, Fatch R, Goodman G, Matthews LT, Magidson JF, et al. High Rates of Biomarker-Confirmed Alcohol Use Among Pregnant Women Living With HIV in South Africa and Uganda. J Acquir Immune Defic Syndr. 2019;82(5):443-51.

12. Cook JL, Green CR, Lilley CM, Anderson SM, Baldwin ME, Chudley AE, et al. Fetal alcohol spectrum disorder: a guideline for diagnosis across the lifespan. CMAJ. 2016;188(3):191-7.

13. Lange S, Probst C, Gmel G, Rehm J, Burd L, Popova S. Global Prevalence of Fetal Alcohol Spectrum Disorder Among Children and Youth: A Systematic Review and Meta-analysis. JAMA Pediatr. 2017;171(10):948-56.

14. Flannigan K, Kapasi A, Pei J, Murdoch I, Andrew G, Rasmussen C. Characterizing adverse childhood experiences among children and adolescents with prenatal alcohol exposure and Fetal Alcohol Spectrum Disorder. Child Abuse Negl. 2021;112:104888.

15. Moore EM, Riley EP. What Happens When Children with Fetal Alcohol Spectrum Disorders Become Adults? Curr Dev Disord Rep. 2015;2(3):219-27.

16. Probst C, Manthey J, Merey A, Rylett M, Rehm J. Unrecorded alcohol use: a global modelling study based on nominal group assessments and survey data. Addiction. 2018;113(7):1231-41. 
17. Probst C, Parry CDH, Rehm J. HIV/AIDS mortality attributable to alcohol use in South Africa: a comparative risk assessment by socioeconomic status. BMJ Open. 2018;8(2):e017955. 18. Joseph Davey D, Kilembe W, Wall KM, Khu NH, Brill I, Vwalika B, et al. Risky Sex and HIV Acquisition Among HIV Serodiscordant Couples in Zambia, 2002-2012: What Does Alcohol Have To Do With It? AIDS Behav. 2017;21(7):1892-903.

19. Kalichman SC, Cain D, Simbayi LC. Multiple recent sexual partnerships and alcohol use among sexually transmitted infection clinic patients, Cape Town, South Africa. Sexually transmitted diseases. 2011;38(1):18-23.

20. Nkosi S, Rich EP, Morojele NK. Alcohol use, sexual relationship power, and unprotected sex among patrons in bars and taverns in rural areas of North West province, South Africa. AIDS and behavior. 2014;18(11):2230-9.

21. Rehm J, Probst C, Shield KD, Shuper PA. Does alcohol use have a causal effect on HIV incidence and disease progression? A review of the literature and a modeling strategy for quantifying the effect. Popul Health Metr. 2017;15(1):4.

22. Zablotska IB, Gray RH, Serwadda D, Nalugoda F, Kigozi G, Sewankambo N, et al. Alcohol use before sex and HIV acquisition: a longitudinal study in Rakai, Uganda. AIDS. 2006;20(8):1191-6.

23. Rehm J, Shield KD, Joharchi N, Shuper PA. Alcohol consumption and the intention to engage in unprotected sex: systematic review and meta-analysis of experimental studies. Addiction. 2012;107(1):51-9. 
24. Shuper PA, Neuman M, Kanteres F, Baliunas D, Joharchi N, Rehm J. Causal considerations on alcohol and HIV/AIDS--a systematic review. Alcohol Alcohol. 2010;45(2):159-

66.

25. Devries KM, Child JC, Bacchus L, Mak J, Falder G, Graham K, et al. Intimate partner violence victimization and alcohol consumption in women: a systematic review and metaanalysis. Addiction. 2014;109(3):379-91.

26. Watt MH, Guidera KE, Hobkirk AL, Skinner D, Meade CS. Intimate partner violence among men and women who use methamphetamine: A mixed-methods study in South Africa. Drug Alcohol Rev. 2017;36(1):97-106.

27. WHO. Intimate Partner IVolence and HIV/AIDS. Geneva, Switzerland: World Health Organization; 2004.

28. O'Connor MJ, Tomlinson M, Leroux IM, Stewart J, Greco E, Rotheram-Borus MJ.

Predictors of alcohol use prior to pregnancy recognition among township women in Cape Town, South Africa. Soc Sci Med. 2011;72(1):83-90.

29. Peltzer K, Mlambo G. Sexual HIV risk behaviour and associated factors among pregnant women in Mpumalanga, South Africa. BMC Pregnancy Childbirth. 2013;13:57.

30. Jones HE, Browne FA, Myers BJ, Carney T, Ellerson RM, Kline TL, et al. Pregnant and nonpregnant women in cape town, South Africa: drug use, sexual behavior, and the need for comprehensive services. Int J Pediatr. 2011;2011:353410.

31. Oldfield BJ, Edelman EJ. Addressing Unhealthy Alcohol Use and the HIV Pre-exposure Prophylaxis Care Continuum in Primary Care: A Scoping Review. AIDS Behav. 2021;25(6):177789. 
32. Williams EC, Hahn JA, Saitz R, Bryant K, Lira MC, Samet JH. Alcohol Use and Human Immunodeficiency Virus (HIV) Infection: Current Knowledge, Implications, and Future Directions. Alcohol Clin Exp Res. 2016;40(10):2056-72.

33. Adeniyi OV, Ajayi Al, Ter Goon D, Owolabi EO, Eboh A, Lambert J. Factors affecting adherence to antiretroviral therapy among pregnant women in the Eastern Cape, South Africa. BMC Infect Dis. 2018;18(1):175.

34. Thomson KA, Hughes J, Baeten JM, John-Stewart G, Celum C, Cohen CR, et al. Increased Risk of HIV Acquisition Among Women Throughout Pregnancy and During the Postpartum Period: A Prospective Per-Coital-Act Analysis Among Women With HIV-Infected Partners. J Infect Dis. 2018;218(1):16-25.

35. Fiebig EW, Wright DJ, Rawal BD, Garrett PE, Schumacher RT, Peddada L, et al. Dynamics of HIV viremia and antibody seroconversion in plasma donors: implications for diagnosis and staging of primary HIV infection. AIDS. 2003;17(13):1871-9.

36. Daar ES, Moudgil T, Meyer RD, Ho DD. Transient high levels of viremia in patients with primary human immunodeficiency virus type 1 infection. N Engl J Med. 1991;324(14):961-4. 37. Heberlein A, Leggio L, Stichtenoth D, Hillemacher T. The treatment of alcohol and opioid dependence in pregnant women. Curr Opin Psychiatry. 2012;25(6):559-64.

38. Joseph Davey DL, Nyemba DC, Gomba Y, Bekker LG, Taleghani S, DiTullio DJ, et al. Prevalence and correlates of sexually transmitted infections in pregnancy in HIV-infected anduninfected women in Cape Town, South Africa. PLoS One. 2019;14(7):e0218349.

39. National Department of Health. Sexually transmitted infections: management guidelines 2018. 2018. 
40. Koen J, Slack C, Barsdorf N, Essack Z. Payment of trial participants can be ethically sound: moving past a flat rate. South African Medical Journal. 2008;98(12):926-9.

41. Babor T, Higgins-Biddle J, Saunders J, Monteiro M. AUDIT: the alcohol use disorders identification test: guidelines for use in primary care. Geneva, Switzerland: World Health Organization; 2001.

42. Babor TF, de la Fuente JR, Saunders J, Grant M. AUDIT. The Alcohol Use Disorders Identification Test. Guidelines for use in primary health care. Geneva, Switzerland: World Health Organization; 1992.

43. May PA, Hasken JM, De Vries MM, Marais AS, Stegall JM, Marsden D, et al. A utilitarian comparison of two alcohol use biomarkers with self-reported drinking history collected in antenatal clinics. Reprod Toxicol. 2018;77:25-32.

44. Vythilingum B, Roos A, Faure SC, Geerts L, Stein DJ. Risk factors for substance use in pregnant women in South Africa. S Afr Med J. 2012;102(11 Pt 1):851-4.

45. Bradley KA, Bush KR, Epler AJ, Dobie DJ, Davis TM, Sporleder JL, et al. Two brief alcoholscreening tests From the Alcohol Use Disorders Identification Test (AUDIT): validation in a female Veterans Affairs patient population. Arch Intern Med. 2003;163(7):821-9.

46. Skagerstrom J, Chang G, Nilsen P. Predictors of drinking during pregnancy: a systematic review. J Womens Health (Larchmt). 2011;20(6):901-13.

47. Myer L, Morroni C, Rebe K. Prevalence and determinants of fertility intentions of HIVinfected women and men receiving antiretroviral therapy in South Africa. AIDS Patient Care STDS. 2007;21(4):278-85. 
48. Cooper D, Moodley J, Zweigenthal V, Bekker LG, Shah I, Myer L. Fertility intentions and reproductive health care needs of people living with HIV in Cape Town, South Africa: implications for integrating reproductive health and HIV care services. AIDS Behav. 2009;13 Suppl 1:38-46.

49. Cox JL, Holden JM, Sagovsky R. Detection of postnatal depression. Development of the 10-item Edinburgh Postnatal Depression Scale. Br J Psychiatry. 1987;150:782-6.

50. Levis B, Negeri Z, Sun Y, Benedetti A, Thombs BD, Group DESDE. Accuracy of the Edinburgh Postnatal Depression Scale (EPDS) for screening to detect major depression among pregnant and postpartum women: systematic review and meta-analysis of individual participant data. BMJ. 2020;371:m4022.

51. Basile $\mathrm{K}$, Hertz $\mathrm{M}$, Black S. Intimate partner violence and sexual violence victimization assessment instruments for use in healthcare settings. . 2007; Version 1.

52. Garcia-Moreno C, Jansen HAFM, Ellsberg M, Heise L, Watts CH. Prevalence of intimate partner violence: findings from the WHO multi-country study on women's health and domestic violence. The Lancet. 2006;368(9543):1260-9.

53. SAS Institute Inc. SAS University Edition. Cary, NC.: SAS Institute Inc. .

54. Moran A, Mashele N, Mvududu R, Gorbach P, Bekker LG, Coates TJ, et al. Maternal PrEP Use in HIV-Uninfected Pregnant Women in South Africa: Role of Stigma in PrEP Initiation, Retention and Adherence. AIDS Behav. 2021.

55. Ernhart CB, Morrow-Tlucak M, Sokol RJ, Martier S. Underreporting of alcohol use in pregnancy. Alcohol Clin Exp Res. 1988;12(4):506-11. 
56. Alvik A, Heyerdahl S, Haldorsen T, Lindemann R. Alcohol use before and during pregnancy: a population-based study. Acta Obstet Gynecol Scand. 2006;85(11):1292-8.

57. Massey SH, Lieberman DZ, Reiss D, Leve LD, Shaw DS, Neiderhiser JM. Association of clinical characteristics and cessation of tobacco, alcohol, and illicit drug use during pregnancy. Am J Addict. 2011;20(2):143-50.

58. Hellerstedt WL, Pirie PL, Lando HA, Curry SJ, McBride CM, Grothaus LC, et al. Differences in preconceptional and prenatal behaviors in women with intended and unintended pregnancies. Am J Public Health. 1998;88(4):663-6.

59. Culley CL, Ramsey T, Mugyenyi G, Kiwanuka G, Ngonzi J, Macleod S, et al. Alcohol exposure among pregnant women in sub-saharan Africa: a systematic review. J Popul Ther Clin Pharmacol. 2013;20(e321-33).

60. Addila AE, Bisetegn TA, Gete YK, Mengistu MY, Beyene GM. Alcohol consumption and its associated factors among pregnant women in Sub-Saharan Africa: a systematic review and meta-analysis' as given in the submission system. Subst Abuse Treat Prev Policy. 2020;15(1):29. 61. Eaton LA, Kalichman SC, Sikkema KJ, Skinner D, Watt MH, Pieterse D, et al. Pregnancy, alcohol intake, and intimate partner violence among men and women attending drinking establishments in a Cape Town, South Africa township. J Community Health. 2012;37(1):208-16.

62. Eggertson L. Stigma a major barrier to treatment for pregnant women with addictions. CMAJ. 2013;185(18):1562.

63. De Jong $M$, George A, Jacobs T. A scoping review of the determinants of foetal alcohol spectrum disorder in South Africa: an intersectional perspective. Health Policy Plan. 2021. 
64. Myers B, Carney T, Browne FA, Wechsberg WM. A trauma-informed substance use and sexual risk reduction intervention for young South African women: a mixed-methods feasibility study. BMJ Open. 2019;9(2):e024776.

65. Stone R. Pregnant women and substance use: fear, stigma, and barriers to care. Health \& Justice. $2015 ; 3(1)$.

66. Watt MH, Eaton LA, Dennis AC, Choi KW, Kalichman SC, Skinner D, et al. Alcohol Use During Pregnancy in a South African Community: Reconciling Knowledge, Norms, and Personal Experience. Matern Child Health J. 2016;20(1):48-55.

67. Sileo K, Miller AP, Huynh TA, Kiene S. A systematic review of interventions for reducing heavy episodic drinking and related outcomes in sub-Saharan African settings. PLOS ONE. 2020. 68. Sileo KM, Miller AP, Wagman JA, Kiene SM. Psychosocial interventions for reducing alcohol consumption in sub-Saharan African settings: a systematic review and meta-analysis. Addiction. 2020.

69. Rotheram-Borus MJ, Tomlinson M, Roux IL, Stein JA. Alcohol Use, Partner Violence, and Depression: A Cluster Randomized Controlled Trial Among Urban South African Mothers Over 3 Years. Am J Prev Med. 2015;49(5):715-25.

70. Rotheram-Borus MJ, Arfer KB, Christodoulou J, Comulada WS, Stewart J, Tubert JE, et al. The association of maternal alcohol use and paraprofessional home visiting with children's health: A randomized controlled trial. J Consult Clin Psychol. 2019;87(6):551-62.

71. Marais S, Jordaan E, Viljoen D, Olivier L, de Waal J, Poole C. The effect of brief interventions on the drinking behaviour of pregnant women in a high-risk rural South African community: a cluster randomised trial. Early Child Development and Care. 2010;181(4):463-74. 
72. Myers BJ, Louw J, Pasche SC. Inequitable access to substance abuse treatment services in Cape Town, South Africa. Subst Abuse Treat Prev Policy. 2010;5:28.

73. UNAIDS. A short technical update on self-testing for HIV. Geneva: Joint United Nations Programme on HIV/AIDS. 2013.

74. Kilembe W, Wall KM, Mokgoro M, Mwaanga A, Dissen E, Kamusoko M, et al. Implementation of couples' voluntary HIV counseling and testing services in Durban, South Africa. BMC Public Health. 2015;15:601.

75. Wall KM, Inambao M, Kilembe W, Karita $E$, Chomba E, Vwalika B, et al. Costeffectiveness of couples' voluntary HIV counselling and testing in six African countries: a modelling study guided by an HIV prevention cascade framework. J Int AIDS Soc. 2020;23 Suppl 3:e25522.

76. World Health Organization. Consolidated guidelines on the use of antiretroviral drugs for treating and preventing HIV infection 2016 [September 16, 2021]. Available from: http://www.who.int/hiv/pub/arv/arv-2016/en/.

77. Joseph Davey DL, Pintye J, Baeten JM, Aldrovandi G, Baggaley R, Bekker LG, et al. Emerging evidence from a systematic review of safety of pre-exposure prophylaxis for pregnant and postpartum women: where are we now and where are we heading? J Int AIDS Soc. 2020;23(1):e25426.

78. Heffron R, Mugo N, Hong T, Celum C, Marzinke MA, Ngure K, et al. Pregnancy outcomes and infant growth among babies with in-utero exposure to tenofovir-based preexposure prophylaxis for HIV prevention. AIDS. 2018;32(12):1707-13. 
medRxiv preprint doi: https://doi.org/10.1101/2021.11.30.21267089; this version posted December 2, 2021. The copyright holder for this preprint (which was not certified by peer review) is the author/funder, who has granted medRxiv a license to display the preprint in perpetuity. It is made available under a CC-BY-NC-ND 4.0 International license.

79. Daniels J, De Vos L, Mogos W, Olivier D, Shamu S, Mudau M, et al. Factors influencing sexually transmissible infection disclosure to male partners by HIV-positive pregnant women in Pretoria townships, South Africa: a qualitative study. Sex Health. 2019;16(3):274-81.

80. Taleghani S, Joseph-Davey D, West SB, Klausner HJ, Wynn A, Klausner JD. Acceptability and efficacy of partner notification for curable sexually transmitted infections in sub-Saharan Africa: A systematic review. Int J STD AIDS. 2019;30(3):292-303. 